\title{
Trade-offs in Primary Detection Using a Mobile Phone as a Sensing Device
}

\author{
Mikko Vääräkangas, Sami Kallioinen*, \\ Aarno Pärssinen* \\ Nokia Research Center, Helsinki, Finland \\ *Currently with Renesas Mobile, Helsinki, Finland
}

\author{
Vesa Turunen, Jussi Ryynänen \\ Aalto University School of Electrical Engineering \\ Department of Micro- and Nanosciences \\ SMARAD CoE \\ Espoo, Finland
}

\begin{abstract}
Cognitive Radio built on top of spectrum sensing is promised to provide more capacity for users wherever and whenever needed and improve radio spectrum utilization in clever and fair manner. However, practical spectrum sensors have to be implemented and they should provide sufficient protection to legacy users of radio spectrum. This leads to trade-offs between primary user protection ratio, device form factor, cost and power consumption. In this research we have derived requirements for antenna efficiency, sensitivity and linearity of practical spectrum sensors. These are verified by field measurements using spectrum sensor embedded to a mobile phone.
\end{abstract}

Keywords; cognitive radio, spectrum sensing, mobile phone, white space devices, digital televison; DVB; UHF; intermodulation

\section{INTRODUCTION}

Cognitive radio network has two ultimate goals to address [1]: First, to provide highly reliable communication for all users of the network, wherever and whenever needed. Second, facilitate efficient utilization of the radio spectrum in a fair-minded and cost-effective manner. Spectrum sensing is key enabler for cognitive radio, allowing every device access to unutilized resource, when and where it is available and needed. However, legacy devices have to be given special rights to radio spectrum when it is justified and well reasoned. For this Federal Communications Commission (FCC) has set rules in the United States of America [2].

In order to protect legacy or primary devices two basic approaches have been presented: geo location database [3] and spectrum sensing. First method needs reliable location of the device and access to the database. Basically there are two different types of devices using the database: fixed devices, like Wi-Fi access points, establishing CR networks and mobile devices, joining available networks. Devices that either do not have connection to the database or exact geo location information are the problem. In first phase of secondary spectrum usage, database based method will be dominant. However, spectrum sensing has several advantages why more research is requested [4].

Initial approach in primary user protection using spectrum sensing was to set fixed, easy to certificate measure: sensitivity. Requirement was set to $-114 \mathrm{dBm}$ in $6 \mathrm{MHz}$ bandwidth. Sensitivity requirement provides an easy way to limit interference level of primary users. Developed spectrum sensing algorithms have been shown to fulfill initial FCC requirements for sensitivity. This problem has two edges: too high primary protection ratio reduces available capacity for secondary usage. Even current sensitivity requirement makes spectrum sensors very hard to implement with state-of-the-art radio front-ends and antennas, especially in small form-factors and power consumption.

Presented CR demonstrators with spectrum sensor use either lab grade measurement instruments with high price, high power consumption, and large form factor, or they does not provide sufficient linearity to avoid problems with strong interfering signals [5]-[8].

Sensitivity of small cheap commercial embedded devices is affected by antenna issues. Spectrum sensor prototype with built-in UHF antenna is described in companion paper [9]. It shows that lower end of TV band suffers from small antennas in mobile phone sized devices. There is also a trade-off between sensitivity and dynamic range of spectrum sensors, especially with wide-band systems [10]. We show by field measurements that either we have to accept severe capacity loss because limited linearity of practical spectrum sensors or we have to develop scheme to relax sensitivity requirement of single sensor. Collaborative sensing via topographical spectrum map provides interesting alternative for calculated geo location database.

\section{SPECTRUM SENSOR PROTOTYPE}

Used spectrum sensor prototype is implemented on Nokia N900 mobile computer [9]. It has following custom parts: built in UHF antenna, UHF receiver, and FFT-based cyclostationary feature detector on FPGA [11]. Needed controls and information gathering mechanics are implemented as SW processes. Gathered information consists of band, channel, received signal strength estimate, feature detector statistics value, date and time, and GPS location if available. Measurement data is collected into database, implemented either on device or into a server on the Internet.

This research is supported by Tekes (the Finnish Funding Agency for Technology and Innovation). 
System performance is presented with details on [9]. Key parameters are shown in TABLE I.

TABLE I. Spectrum Sensor Prototype Performance

\begin{tabular}{|l|c|}
\hline Frequency range & $570-850 \mathrm{MHz}$ \\
\hline Bandwidth & $8 \mathrm{MHz}$ \\
\hline $\mathrm{NF}$ & $4 \mathrm{~dB}$ \\
\hline IIP3 & $-11 \mathrm{dBm}$ \\
\hline Antenna efficiency & $-14-(-7) \mathrm{dB}$ \\
\hline Detection time & $460 \mathrm{~ms}$ \\
\hline Sensitivity, Pdet $=0.9$ & $-118 \mathrm{dBm}$ \\
\hline False alarm rate & 0.01 \\
\hline
\end{tabular}

\section{FIELD MEASUREMENTS}

Two sets of field tests were carried out in capital area in Finland. First measurement set was done mostly outdoors in urban Ruoholahti area in Helsinki. Two sensors were used, both using internal antennas. The measurement set consists of spectral samples from 37 different locations, shown in Fig. 1. One spectral sample includes detection time, GPS location, band, channel, received signal strength in $\mathrm{dBm}$ and DVB-T detection statistics from UHF channels 34 to 60 (578 - $784 \mathrm{MHz}$ ). Detection time was set to $460 \mathrm{~ms}$ and detection statistics positive detection threshold to produce constant false alarm rate of $1 \%$. Measured signal strengths are shown in Fig. 2. Corresponding estimated probabilities of DVB-T detections on different channels are shown in Fig. 3. There is DVB-H repeater in the area, detected on channel 35. Espoo TV transmitter is transmitting on channels $32,35,44,46$, and 53.

TABLE II. DVB-T TRANSMITTER PARAMETERS.

\begin{tabular}{|l|c|c|}
\hline \multicolumn{1}{|c|}{ DVB-T transmitters } & Espoo & Tallinn \\
\hline Latitude: & 60.1778 & 59.4713 \\
\hline Lognitude: & 24.6403 & 24.8875 \\
\hline Mast heigth: & $313 \mathrm{~m}$ & $272 \mathrm{~m}$ \\
\hline Transmission power: & $47 \mathrm{dBm}$ & $42 \mathrm{dBm}$ \\
\hline Occupied channels & $32,35,44,46,53$ & $45,59,64$ \\
\hline
\end{tabular}

TV transmissions on measurement range are detected with high probability. Channel 59 is occupied by Tallinn TV transmitter on average $78 \mathrm{~km}$ away. Open source Splat! [12] radio propagation calculation tool, using Longley-Rice Irregular Terrain Model [13] and NASA SRTM-3 Version 2 Elevation Models [14], was used for field strength estimation. Used transmitter parameters are shown in TABLE II. , receiver was assumed to be $3 \mathrm{~m}$ above sea surface. Estimated field strength, shown in Fig. 4, in Ruoholahti area is $20-60 \mathrm{~dB} \mu \mathrm{V} / \mathrm{m}$. Field strength has large variation within $1 \mathrm{~km}$ radius in urban area. Relationship between field strength and received signal power at the antenna connector is following [15]:
$S(d B m)=20 \log (f(M H z))+77.2-E\left(\frac{d B \mu V}{m}\right)+G(d B)$

Where $S(\mathrm{dBm})$ is signal power at the antenna connector in $\mathrm{dBm}, f(M H z)$ is used frequency in $\mathrm{MHz}$ and $\mathrm{E}(\mathrm{dB} \mu \mathrm{V} / \mathrm{m})$ is field strength in $\mathrm{dB} \mu \mathrm{V} / \mathrm{m}$, and $G(d B)$ is antenna gain.

If antenna gain is taken into account, efficiency and directivity should be analyzed carefully. With mobile devices, we are interested in average gain, not peak gain, because device alignment is random. Another aspect is that in most of the cases signal is not arriving directly via line of sight channel, but rather via multipath route and peak gain or minimum gain are not interesting when average performance of sensing system is studied. Therefore antenna is assumed to have isotropic radiation pattern on average with measured antenna efficiency.

With measured prototype antenna, having antenna efficiency of $-7.5 \mathrm{~dB}$, simulated field strength corresponds $-123-(-83) \mathrm{dBm}$ signal input power at the receiver. Taking measured detector sensitivity into account we end up 0.6 to 1 detection probability of Tallinn TV transmitter in Ruoholahti, Helsinki. Tallinn transmission on channel 45, adjacent to much stronger Espoo TV transmitter on channel 44 and 46, is masked and it cannot be detected. One must remember that transmissions from Tallinn are out of the reach for typical TV reception setups in Helsinki households.

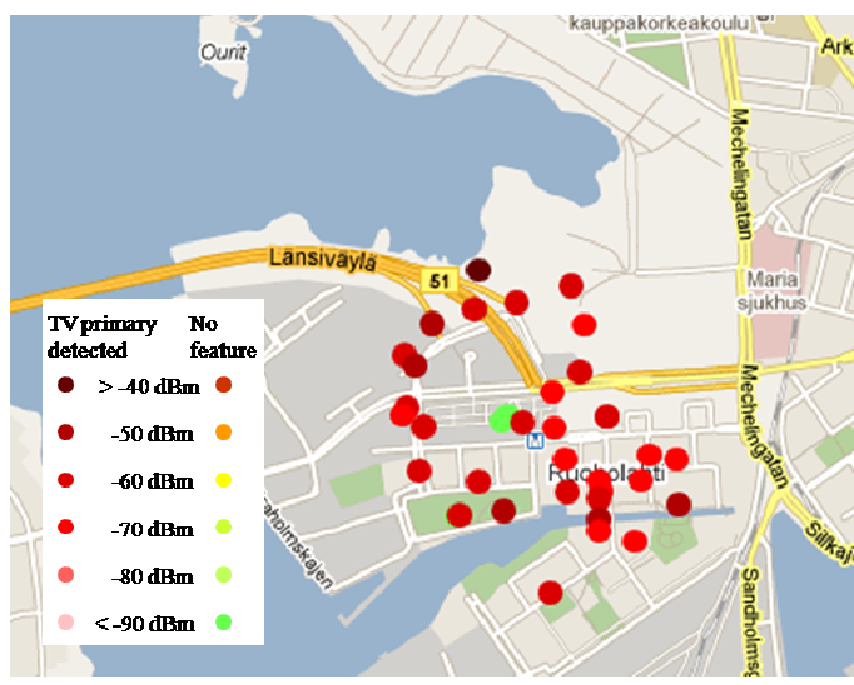

Fig. 1. Measurements results on UHF channel $44(658 \mathrm{MHz})$ in Ruoholahti, Helsinki.

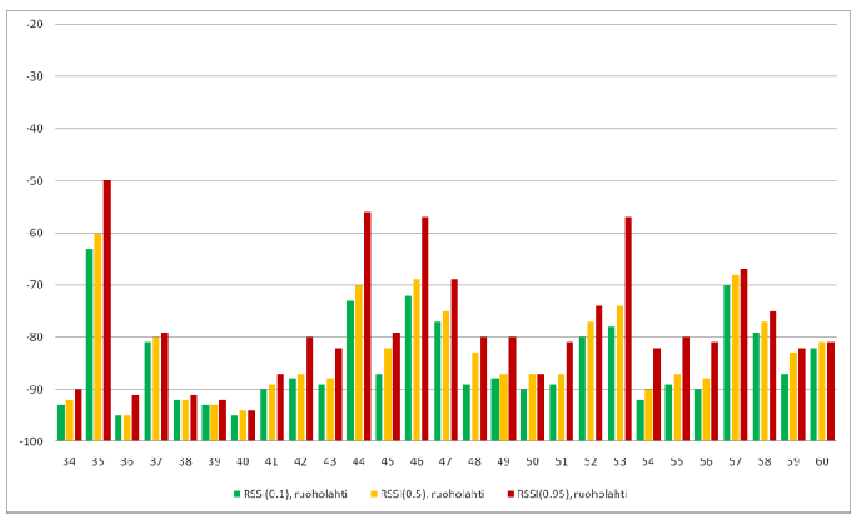


Fig. 2. Received signal strength (RSSI $[\mathrm{dBm}]$ ) upper limit for $10 \%, 50 \%$ and $95 \%$ of measured samples in Ruoholahti.

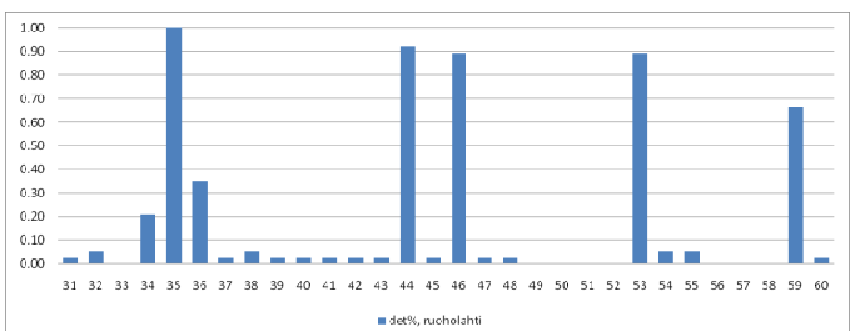

Fig. 3. Measured probability of DVB-T detection, $n=37$ per UHF channel, average distance to Espoo transmitter $15 \mathrm{~km}$ and $78 \mathrm{~km}$ to Tallinn transmitter.

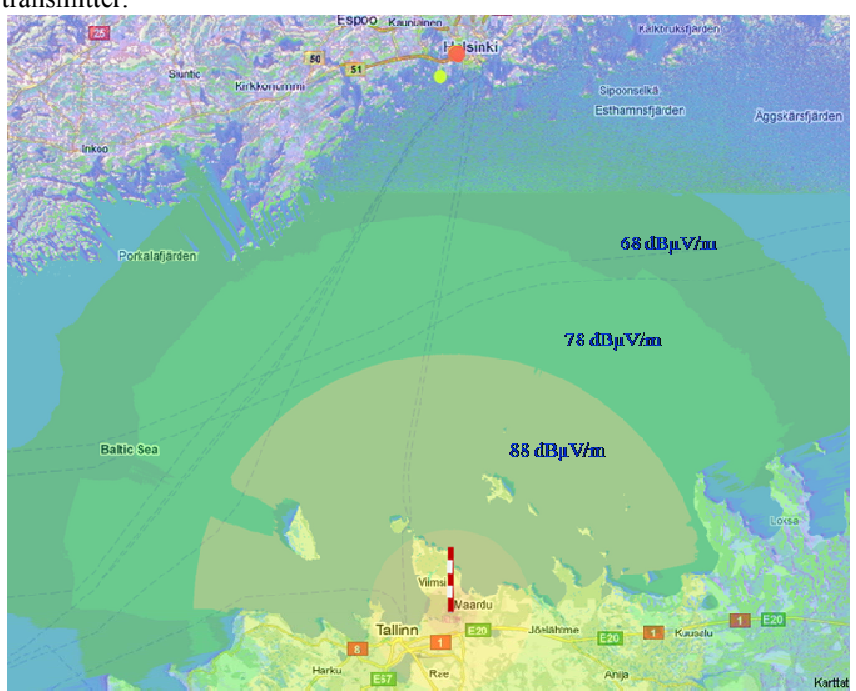

Fig. 4. Simulated field strengths on UHF channel $59(778 \mathrm{MHz})$ from Tallinn TV tower, distance to Helsinki $77 \mathrm{~km}$.

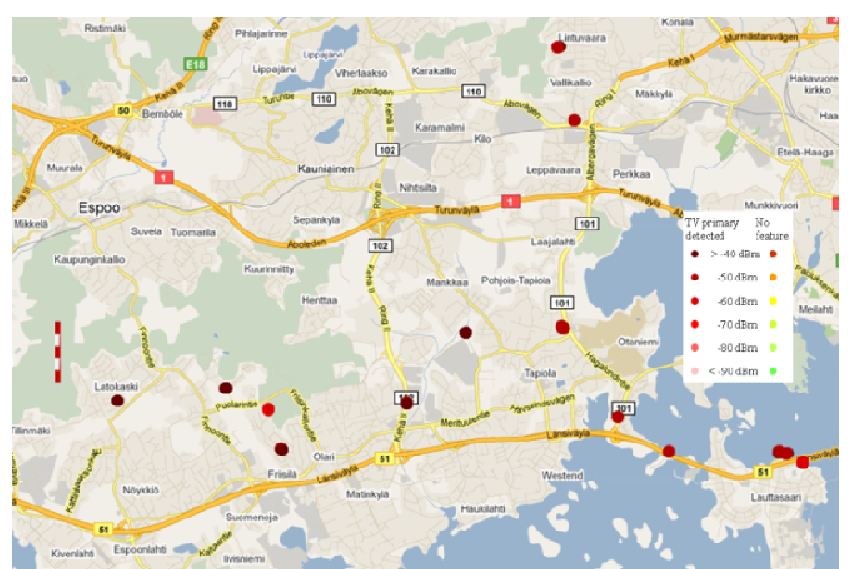

Fig. 5. Measurements results on UHF channel $44(658 \mathrm{MHz})$ in Espoo, average distance to Espoo TV tower is $8 \mathrm{~km}$.

Second set was measured outdoors in suburban Espoo and target was to evaluate performance of the spectrum sensor in the vicinity of strong TV transmitter. Measurements were done using two sensors one with internal antenna and another with external reference dipole. Measurement locations and results for occupied channel 44 $(658 \mathrm{MHz})$ are shown in Fig. 5. Measured signal power on occupied channels was from $-65 \mathrm{dBm}$ up to $-32 \mathrm{dBm}$, when using external reference dipole, as shown in Fig. 6. The $\mathrm{RSSI}[\mathrm{dBm}]$ limits tell how many percent of measurement samples have smaller RSSI value than presented. Basically this presents values of observed cumulative distribution function with $10 \%, 50 \%$ and $95 \%$ probabilities. TV signal strength is from 10 to $30 \mathrm{~dB}$ more than in the Helsinki measurement set. Main difference between results measured using internal and external antennas is that antenna efficiency of internal antenna is on average $7.5 \mathrm{~dB}$ lower than external reference dipole. Results using internal antenna are shown in Fig. 7. Difference in antenna efficiency means additional noise figure of $7.5 \mathrm{~dB}$, which decreases sensitivity and increases linearity of receiver. In addition there is also device induced noise in internal antenna measurements. Performance difference is clearer with detection results shown in Fig. 8.

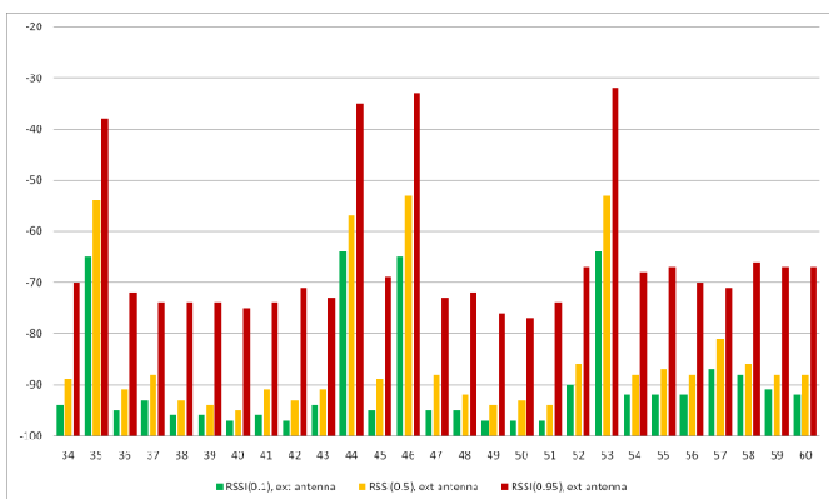

Fig. 6. Received signal strength (RSSI $[\mathrm{dBm}]$ ) with external antenna, upper limit for $10 \%, 50 \%$ and $95 \%$ of measured samples in Espoo.

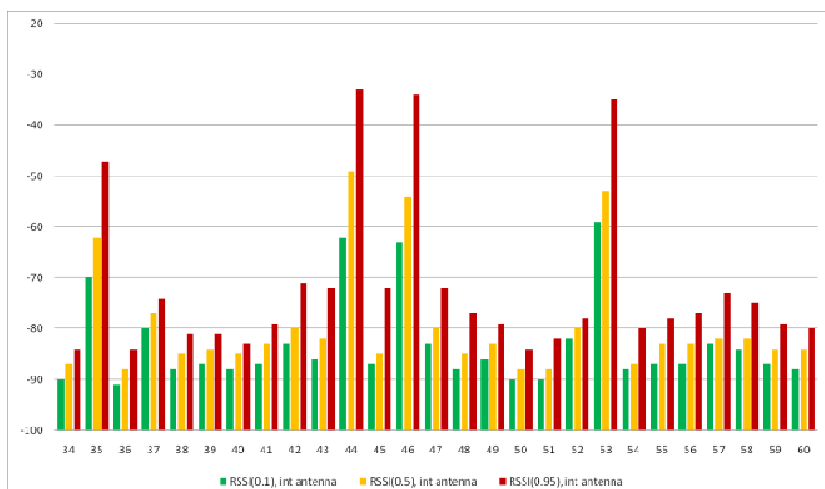

Fig. 7. Received signal strength (RSSI $[\mathrm{dBm}]$ ) with internal antenna, upper limit for $10 \%, 50 \%$ and $95 \%$ of measured samples in Espoo.

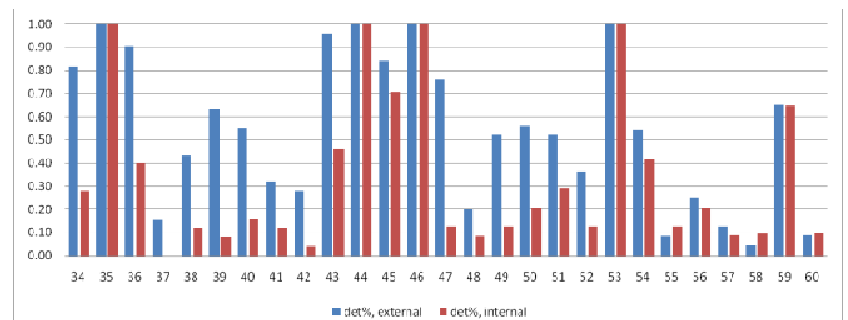

Fig. 8. Measured probability of DVB detection, number of detections is 26 per channel, average distance to Espoo transmitter $9 \mathrm{~km}$ and $78 \mathrm{~km}$ to Tallinn transmitter. 


\section{INTERMODULATION RESULT ANALYSIS}

Memoryless weakly non-linear system model is videly used for modeling radio front-end nonlinear phenomena.

$$
\mathrm{S}_{\text {out }}=k_{1} \mathrm{~S}_{\text {in }}+k_{2} \mathrm{~S}_{\text {in }}^{2}+k_{3} \mathrm{~S}_{\text {in }}^{3}
$$

Equation (1) is based on trunked Taylor series expansion. Almost always $k_{0}$ term i.e. DC offset is removed in receiver and it is not shown here. The first term with $k_{1}$ is the amplified version of $\mathrm{Sin}$. The second and the third term will be called the 2 nd order and 3 rd order nonlinear terms, respectively. Those nonlinear terms generate different distortion products. Some of those distortion products fall into the band of interest. It is often assumed that UHF TV band selection filter can handle the second order term [10]. However, we show that this will not be the case with modulated signal, e.g. DVB. The effect of the 3 rd order nonlinear terms cannot be ignored because their distortion products always fall inside the desired DTV spectrum.

In case of Espoo transmitter, DVB-T signals on channels $32,35,44,46$, and 53 we get input signal

$$
\mathrm{S}_{\text {in }}=\operatorname{Re}\left\{\sqrt{2} \sum_{n=32,35,44,46,53} A_{t v, n} e^{i \omega_{n}}\right\}
$$

Power level of intermodulation results can be calculated as follows

$$
\begin{gathered}
P_{I M 3}=3 P_{i n}-2 I I P_{3} \\
P_{I M 2}=2 P_{i n}-I I P_{2}
\end{gathered}
$$

By using (2), (3), (4), wideband signal effect ( $\left.\Delta_{\text {wideband }}\right)$, and spectrum spreading with IM3 into account $(66 \%$ on the channel and $17 \%$ to adjacent channels) [17], we get IM3 simulation results shown in Fig. 9. Calculated result is very similar than measured, that is shown in Fig. 6. IM2 result in (5) is used to derive IIP $_{2}$ requirement of spectrum sensor.

Spectrum sensor performance with single strong DVB transmission is verified by laboratory measurements. Signal is placed on channel 45 and detection probability is measured from channels 45, 46, 47, and 48. Results are shown in Fig. 10. Results measured on channel 45 shows the sensitivity of the prototype detector. DVB signal is detected from adjacent channel if its power is over $-60 \mathrm{dBm}$ and over $-45 \mathrm{dBm}$ signals can be seen even more than 4 channels away. Baseband filter bandwidth is set to $5 \mathrm{MHz}$, the same than used in field measurements. In addition, signal is oversampled by a factor of 4 and digital filtering attenuates adjacent channel signal down to quantization noise before the detector, thereby excluding aliasing effects from the neighboring channels. It is quite obvious that a seen phenomenon comes from second order intermodulation of the sensor front-end. Most likely from self-mixing of modulated signal.

In order to guarantee enough capacity for sensing devices, IIP3 and IIP2 requirement for spectrum sensor should be specified. To avoid false detections due to IM products, IIP3 and IIP2 requirements should be set so that resulting IM products power will be under constant false alarm threshold. In case of used cyclostationary feature detector, this level is $-124 \mathrm{dBm}$. In our field measurements $95 \%$ of detected signal levels were under $-30 \mathrm{dBm}$, corresponding requirements for IIP 3 of $17 \mathrm{dBm}$, and IIP2 of $64 \mathrm{dBm}$ with external dipole antenna. With built-in UHF antenna, same requirements are $5.7 \mathrm{dBm}$ IIP3 and $49 \mathrm{dBm}$ IIP2. One should remember that the prototype meet FCC sensitivity requirements only in upper part of UHF TV band, because antenna efficiency is very poor in lower part. How cyclostationary features will behave in non-linear spectrum sensor front-ends should be investigated more and this approach could provide interesting methods to avoid problems with IM products.

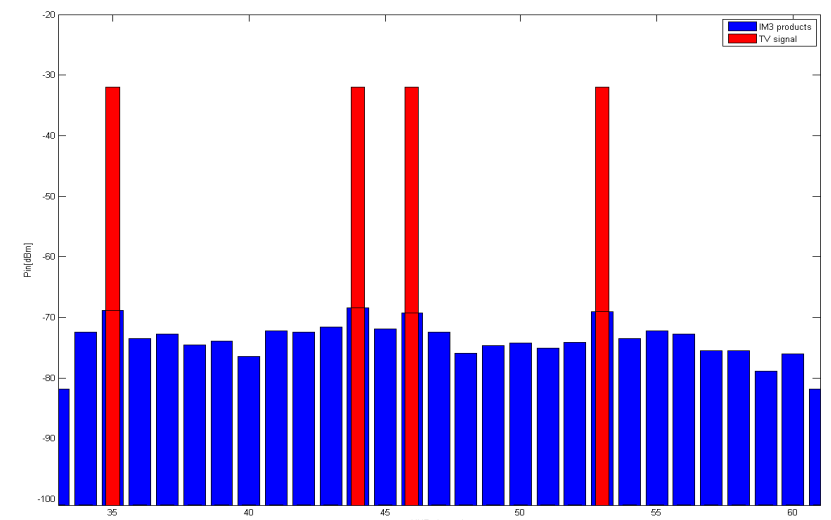

Fig. 9. Calculated power level of IM3 products, IIP3 $=-10$, in case of five $32 \mathrm{dBm}$ DVB-T signals on UHF channels 32, 35, 44, 46, and 53.

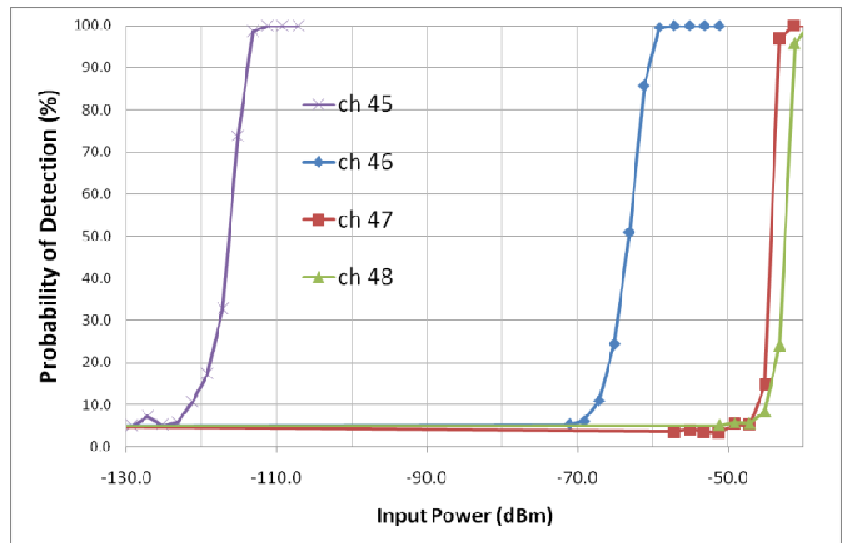

Fig. 10. Adjacent channel measurement results: DVB signal transmitted on channels 45, measured detection probabilities from channels 45, 46, 47, and 48.

\section{CONCLUSIONS}

We have shown by field measurements that single device single snapshot detection is too unreliable due antenna gain minima, interference, and fading to protect primary users. Just tightening of sensitivity requirement does not help, because IM products cause desensitization, masking, and false alarms, thus reducing available capacity for secondary 
users [18]. Methods like geo location databases and using collaborative sensing are developed to solve these problems. Collaborative sensing averages antenna gain and radio propagation problem. However presented trade-offs between sensitivity in sensor linearity requirement and sensitivity has to be taken into account in algorithm development. Collaborative use of network of large amount of cheap spectrum sensors embedded to e.g. mobile phones is interesting and practical approach to solve spectrum sensing problem. Estimation of multi-dimensional spectrum utilization field, using compressive sensing techniques in collaborative spectrum sensing network is interesting approach for enabling truly cognitive radios.

\section{REFERENCES}

[1] Haykin, S.; Thomson, D.J.; Reed, J.H.; , "Spectrum Sensing for Cognitive Radio," Proceedings of the IEEE, vol.97, no.5, pp.849877, May 2009

[2] FCC, "Second Report and Order and Memorandum Opinion and Order," ET Docket No. 08-260, Nov. 2008.

[3] Gurney, D.; Buchwald, G.; Ecklund, L.; Kuffner, S.L.; Grosspietsch, J.; , "Geo-Location Database Techniques for Incumbent Protection in the TV White Space," New Frontiers in Dynamic Spectrum Access Networks, 2008. DySPAN 2008. 3rd IEEE Symposium on, vol., no., pp.1-9, 14-17 Oct. 2008

[4] FCC News, "FCC Crafts Proposals to Boost Spectrum Research and Spectrum Efficient Wireless Technologies,", Nov. 2010. http://www.fcc.gov/Daily_Releases/Daily_Business/2010/db1130/DO C303096A1.pdf

[5] Kai Hong; Sengupta, S.; Chandramouli, R.; , "SpiderRadio: An Incumbent Sensing Implementation for Cognitive Radio Networking Using IEEE 802.11 Devices," Communications (ICC), 2010 IEEE International Conference on, vol., no., pp.1-5, 23-27 May 2010

[6] Kihong Kim; Junki Min; Sungho Hwang; Seongsoo Lee; Kyungseok Kim; Haksun Kim; , "A CR Platform for Applications in TV Whitespace Spectrum," Cognitive Radio Oriented Wireless Networks and Communications, 2008. CrownCom 2008. 3rd International Conference on , vol., no., pp.1-6, 15-17 May 2008

[7] Ser Wah Oh; Naveen, A.A.S.; Yonghong Zeng; Kumar, V.P.; Le, T.P.C.; Kua, K.; Weiqiang Zhang; , "White-Space Sensing Device for Detecting Vacant Channels in TV Bands," Cognitive Radio Oriented Wireless Networks and Communications, 2008. CrownCom 2008. 3rd International Conference on , vol., no., pp.1-6, 15-17 May 2008

[8] Hur, Y.; Park, J.; Kim, K.; Lee, J.; Lim, K.; Lee, C.-H.; Kim, H.S.; Laskar, J.; , "A Cognitive Radio (CR) Testbed System Employing a Wideband Multi-Resolution Spectrum Sensing (MRSS) Technique," Vehicular Technology Conference, 2006. VTC-2006 Fall. 2006 IEEE 64th , vol., no., pp.1-5, 25-28 Sept. 2006

[9] Kallioinen, S.; Vääräkangas, M.; Hui, P.; Ollikainen, J.; Teikari, I.; Pärssinen, A.; Turunen, V.; Kosunen, M; Ryynänen, J. "Multi-Mode Multi-Band Spectrum Sensor for Cognitive Radios Embedded to a Mobile Phone", accepted to be published in 6th International ICST Conference on Cognitive Radio Oriented Wireless Networks (CrownCom), 1-3 June 2011

[10] N. A. Moseley, E. A. M. Klumperink, and B. Nauta, “A spectrum sensing technique for cognitive radios in the presence of harmonic images," in Proc. 3rd IEEE Symp. on New Frontiers in Dynamic Spectrum Access Networks (DySPAN), 2008, pp. 1-10

[11] V. Turunen, M. Kosunen, A. Huttunen, S. Kallioinen, P. Ikonen, A. Pärssinen and J. Ryynänen, "Implementation of cyclostationary feature detector for cognitive radios," in Proc. Int. Conf. Cognitive Radio Oriented Wireless Networks and Communications, 2009, pp. 14.

[12] http://www.qsl.net/kd2bd/splat.html

[13] http://flattop.its.bldrdoc.gov/itm.html

[14] http://www2.jpl.nasa.gov/srtm/
[15] Q. Gu, "RF System Design of Transceivers for Wireless Communications", Spriger Science+Business Media, 2005

[16] M. Alink, E. Klumperink, M. Soer, A. Kokkeler and B. Nauta, "A 50MHz-to-1.5GHz Cross-Correlation CMOS Spectrum Analyzer for Cognitive Radio with $89 \mathrm{~dB}$ SFDR in $1 \mathrm{MHz}$ RBW", in Proc. 4th IEEE Symp. on New Frontiers in Dynamic Spectrum Access Networks (DySPAN), 2010

[17] D. H. Mahrof, E. A. M. Klumperink, J. C. Haartsen, and B. Nauta, "On the effect of spectral location of interferers on linearity requirements for wideband cognitive radio front ends," in Proc. 4th IEEE Symp. on New Frontiers in Dynamic Spectrum Access Networks (DySPAN), 2010

[18] Dongliang Duan; Liuqing Yang; Principe, J.C., "Detectioninterference dilemma for spectrum sensing in cognitive radio systems," Military Communications Conference, 2009. MILCOM 2009. IEEE, vol., no., pp.1-7, 18-21 Oct. 2009 